\title{
Tuberculosis related Neuroretinitis and Retinal Vasculitis in an Immunocompetent Patient
}

\author{
Neha Goel ${ }^{\text {DQ } 8}$ \\ ICARE Eye Hospital and Postgraduate Institute, India
}

\begin{abstract}
Introduction: This study aimed to describe the occurrence of bilateral neuroretinitis and retinal vasculitis in an immunocompetent female with disseminated tuberculosis.

Case description: A 25 year old female diagnosed with disseminated tuberculosis presented with sudden painful loss of vision bilaterally. On examination she was found to have bilateral neuroretinitis and retinal periphlebitis involving the posterior pole. She was prescribed systemic steroids in addition to the antituberculous therapy that she was receiving. The fundus lesions gradually resolved over four months with modest improvement in visual acuity. There were no recurrences in the follow up period of 14 months.

Conclusions: Ocular involvement in the form of neuroretinitis with concomitant retinal vasculitis can occur in patients with disseminated tuberculosis. Addition of systemic steroids to anti-tuberculous therapy leads to anatomical resolution with improvement in visual acuity.

Key words: Neuroretinitis, Ocular tuberculosis, Periphlebitis, Retinal vasculitis.
\end{abstract}

\section{INTRODUCTION}

Intraocular tuberculosis (TB) can mimic various uveitis entities. The commonest clinical presentation has been seen to be posterior uveitis, followed by anterior uveitis, panuveitis, and intermediate uveitis (Gupta V et al, 2007). While periphlebitis is a common manifestation of intraocular TB, optic nerve involvement in the form of neuroretinitis, papilledema, papillitis, optic neuritis, retrobulbar neuritis or optic nerve tubercle is relatively rare or limited to patients with tuberculous meningitis. It may be caused by direct infection or indirect immune-mediated hypersensitivity (Gupta V et al, 2007; Davis EJ et al, 2012). This report describes the clinical course of bilateral neuroretinitis and retinal vasculitis in an immunocompetent female with disseminated TB on anti-tuberculous therapy (ATT).

$\begin{array}{ll}\text { Financial Interest } \quad: \text { Nil } & \text { Received : 15.09.2020 } \\ \text { Conflict of Interest : Nil } & \text { Accepted : 12.03.2021 } \\ \text { Corresponding Author } & \\ \text { Dr. Neha Goel } & \\ \text { ICARE Eye Hospital and Postgraduate Institute, } \\ \text { New Delhi, India. } \\ \text { E-mail: nehadoc@hotmail.com }\end{array}$

Access this article online

Website: www.nepjol.info/index.php/NEPJOPH DOI: https://doi.org/10.3126/nepjoph.v13i2.31212 Copyright $\odot 2021$ Nepal Ophthalmic Society ISSN: 2072-6805, E-ISSN: 2091-0320

This work is licensed under a Creative Commons Attribution-NonCommercial-NoDerivatives 4.0 International License (CC BY-NC-ND). 


\section{CASE DESCRIPTION}

A 25 year old female complained of sudden bilateral vision loss for a day, preceded by pain and fever for a week. She was being evaluated for weight loss for the last three months, which revealed right upper lobe nodulo-infiltrative lesion, mediastinal lymphadenopathy and white matter edema in left temporo parietooccipital region. There was no evidence of raised intracranial pressure. She was diagnosed as disseminated TB and started on standard four drug ATT consisting of rifampicin 600 $\mathrm{mg}$ once daily, isoniazid $450 \mathrm{mg}$ once daily, pyrazinamide $1.5 \mathrm{~g}$ once daily and ethambutol
$800 \mathrm{mg}$ once daily one week back. She was HIV negative.

Her best-corrected visual acuity (BCVA) was perception of light with accurate projection of rays in the right eye and counting fingers close to face in the left eye. Anterior segment examination showed sluggishly reacting pupils in both eyes. Intraocular pressures (IOP) were $12 \mathrm{~mm} \mathrm{Hg}$ and $14 \mathrm{~mm} \mathrm{Hg}$ in the right and left eyes respectively. There was mild vitritis in both eyes. Fundus examination showed bilateral disc edema, retinal vasculitis involving the veins of the posterior pole and incomplete macular star formation, more severe in the left eye (Figure 1a, b).
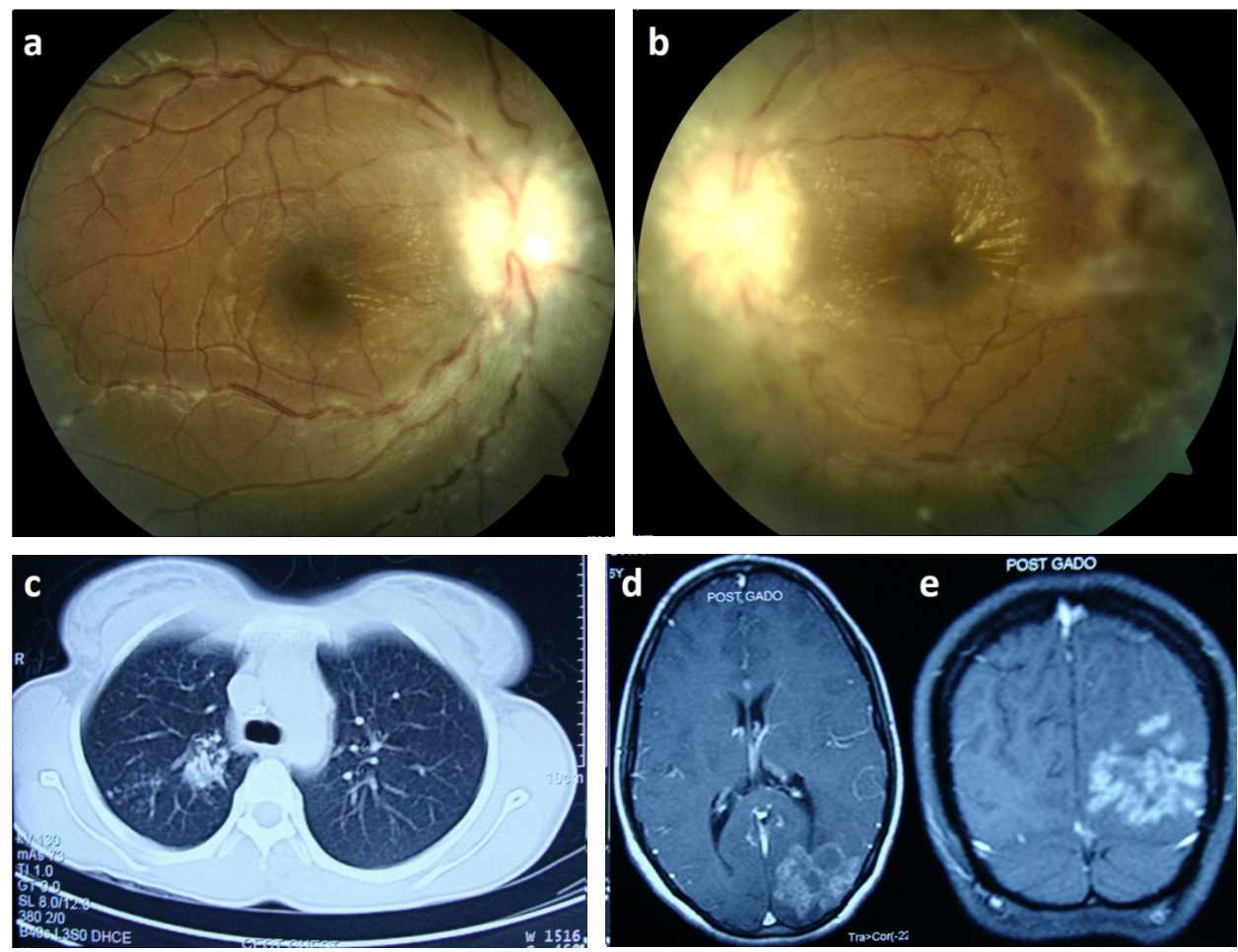

Figure 1: Color fundus photograph of the right (a) and left (b) eye at presentation showing disc edema, vasculitis involving the retinal veins at the posterior pole and incomplete macular star formation (c) Contrast enhanced CT scan of the chest depicting infiltrative and nodular lesions in right upper lobe with calcified mediastinal and right hilar lymph nodes (d) MRI brain demonstrating multiple nodular and peripherally enhancing conglomerate lesions in the left posterior parietal and occipital cortex with hypointense centre on T2 weighted images with moderate perilesional edema, suggestive of tuberculomas. 
A diagnosis of disseminated TB (Figure 1c, d) with bilateral neuroretinitis and retinal vasculitis was made. Under supervision of the physician, systemic steroids (1 $\mathrm{mg} / \mathrm{kg} /$ day $)$ were also prescribed 3 days after presentation. Gradual reduction of disc edema and vasculitis was noted two weeks after initiation of treatment (Figure 2a, b), and BCVA improved to counting fingers close to face in the right eye and counting fingers at 3 meters in the left eye. After 2 months, ATT was switched to rifampicin and isoniazid for 10 months. Steroids were tapered by $10 \mathrm{mg}$ weekly to a maintenance dose of $10 \mathrm{mg}$ per day. At 4 months follow up, BCVA improved further to counting fingers at 3 meters in the right eye and 20/200 in the left eye. Fundus revealed bilateral disc pallor, resolution of the vasculitis and macular star and pre-retinal membranes in the left eye (Figure 2c, d).
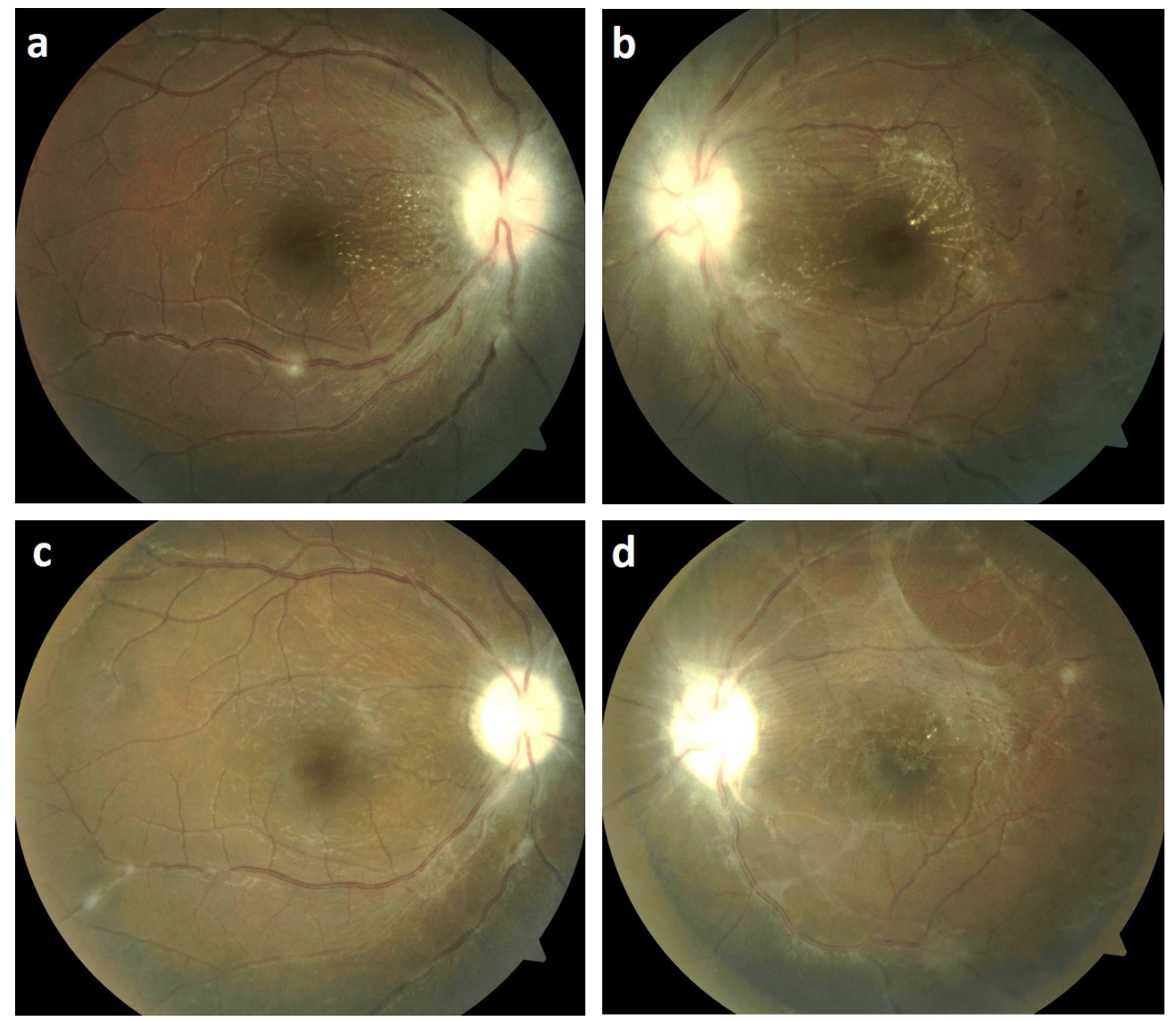

Figure 2: Color fundus photograph of the right (a) and left (b) eye at two weeks following initiation of systemic steroids, in addition to anti-tuberculous therapy. There was reduction in disc edema and retinal vasculitis. At four months follow up, colour fundus photographs of the right (c) and left eye (d) revealed disc pallor and resolution of the vasculitis and macular star. In addition, there were pre-retinal membranes in the left eye at the posterior pole. 

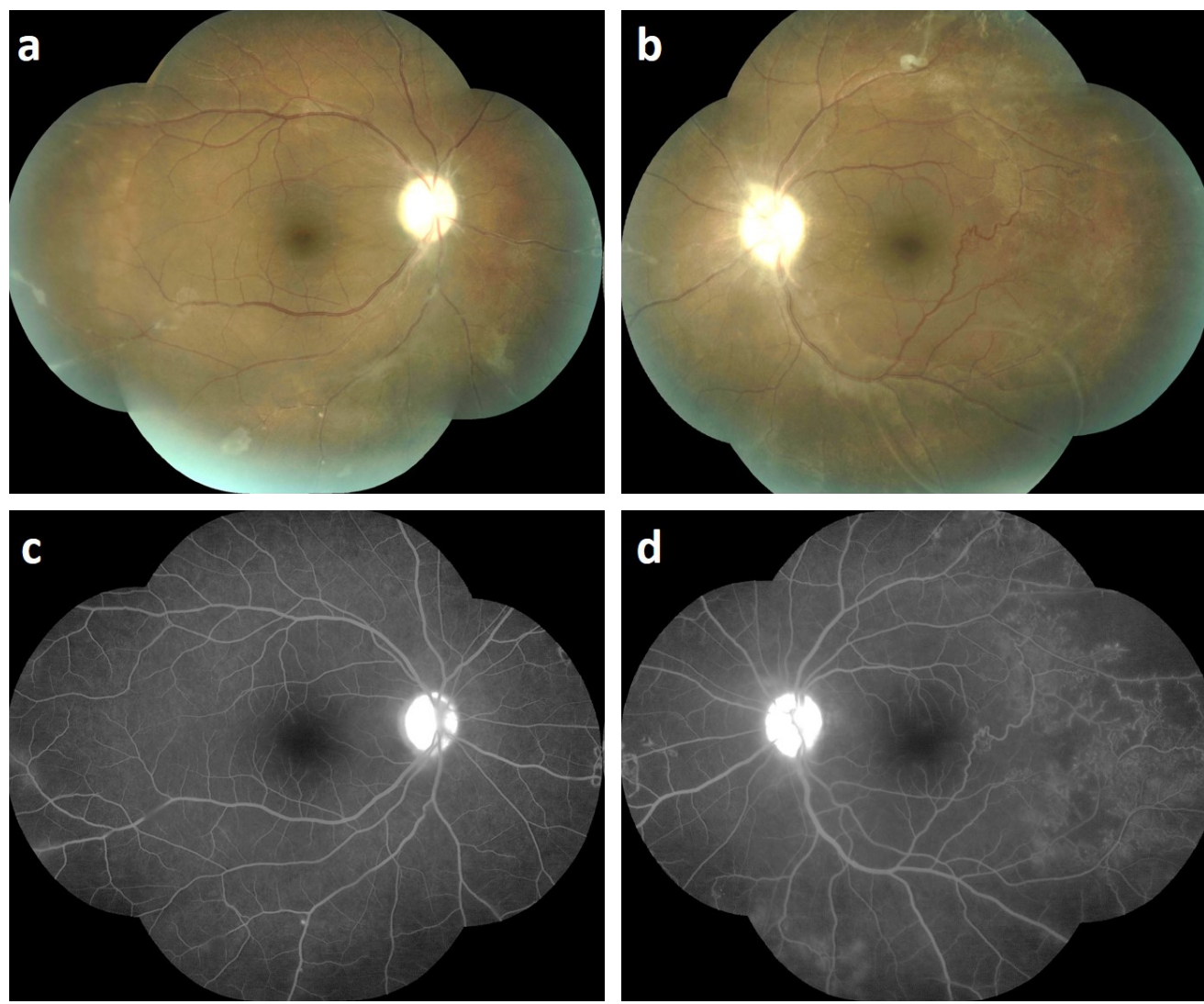

Figure 3: Color fundus photograph of the right (a) and left (b) eye at 14 months follow up showing disc pallor with healed vasculitis and collateral formation. Fundus fluorescein angiography (FFA) of the right eye was unremarkable (c). The left eye showed capillary non perfusion areas temporally and neovascularization inferonasally (d).

Following completion of ATT at 14 months follow up BCVA was 20/200 in the right eye and $20 / 120$ in the left eye. There was bilateral disc pallor with healed vasculitis and collateral formation on fundus examination (Figure $3 a, b$ ). Fundus fluorescein angiography (FFA) showed capillary non perfusion areas temporally and neovascularization inferonasally in the left eye, in addition, which were lasered (Figure $3 \mathrm{c}, \mathrm{d}$ ).

\section{DISCUSSION}

Disseminated TB signifies involvement of two or more non-contiguous organs in a single patient (Iseman MD 2000). This term is preferable to the earlier "miliary TB" since miliary shadowing of the lungs occurs only in a minority of these patients and lacks specificity for disseminated TB (Talbot EA et al, 1999). While majority patients with disseminated TB 
harbour an underlying immunosuppressive condition, most commonly HIV infection, upto $17 \%$ may have no apparent immune disorder (Crump JA et al, 2003). Ocular involvement in disseminated TB results from hematogenous spread of the tuberculous organisms from other primary infectious foci. Single or multiple choroidal tubercles in the absence of anterior and intermediate segment inflammation have been described to be specific for disseminated TB (Mehta S, 2017; Mehta S, 2004).

The above patient was a known case of disseminated TB on ATT. Thus even in the absence of definitive tissue diagnosis, the ocular involvement in the form of bilateral neuroretinitis and retinal vasculitis was presumed to be due to tuberculosis. This presentation in an immunocompetent patient with disseminated TB has not been described previously (Davis EJ et al, 2012; Mehta S, 2017; Mehta S, 2004). Addition of systemic steroids to the ATT resulted in gradual resolution of the bilateral disc edema and retinal vasculitis with partial restoration of visual acuity, with no recurrences till the end of follow up.

Clinical data analysis of 62 eyes of 49 patients with tuberculous optic neuropathy showed papillitis to be the most common involvement, followed by neuroretinitis and optic nerve tubercle (Davis EJ et al, 2012). Posterior uveitis was frequently associated in these patients. Presentation was typically unilateral with painless loss of vision, unlike the above case. None of the patients in this series were described as having disseminated TB though the authors did highlight the importance of investigating for pulmonary or meningeal TB when there is involvement of the optic nerve. More than half of these patients received oral steroids along with ATT and visual outcomes were generally good. The above case showed anatomical resolution with ATT and steroids though functional outcome was suboptimal owing to severe optic nerve involvement.

\section{CONCLUSION}

To conclude, this report illustrates widespread involvement by tubercle bacilli (eye, lungs, brain and lymph nodes) in an immunocompetent patient with disseminated TB. Bilateral neuroretinitis with retinal vasculitis as seen in this case has not been described previously in this setting. Multisystem involvement necessitates a multi-disciplinary approach to therapy. Use of corticosteroids concomitantly with ATT can lead to resolution of the lesions with improvement in visual acuity though visual prognosis may be guarded due to optic neuropathy. A high index of suspicion in endemic areas is important for timely diagnosis and appropriate management. 


\section{REFERENCES}

Gupta V, Gupta A, Rao NA (2007). Intraocular tuberculosis--an update. Surv Ophthalmol;52: 561-87. doi: 10.1016/j. survophthal.2007.08.015; PMid:18029267

Davis EJ, Rathinam SR, Okada AA, Tow SL, Petrushkin H, Graham EM, et al (2012). Clinical spectrum of tuberculous optic neuropathy. J Ophthalmic Inflamm Infect;2:183-9. doi: 10.1007/s12348-012-0079-5; PMid:22614321

Iseman MD (2000). Extrapulmonary tuberculosis in adults. In: A clinician's guide to tuberculosis. 1st ed. Philadelphia: Lippincott Williams \&Wilkins:145-97.

Talbot EA, Reller LB, Frothingham R (1999). Bone marrow cultures for the diagnosis of mycobacterial and fungal infections in patients infected with the human immunodeficiency virus. Int J Tuberc Lung Dis;3:908-12.

Crump JA, Reller LB (2003). Two decades of disseminated tuberculosis at a university medical center: the expanding role of mycobacterial blood culture. Clin Infect Dis;37:1037-43. doi: 10.1086/378273; PMid:14523767

Mehta S (2017). Patterns of ocular inflammation in patients with miliary tuberculosis. F1000Res;6:412. doi: 10.12688/ f1000research.11035.1; PMid:28503298

Mehta S (2004). Ocular lesions in acute disseminated tuberculosis. Ocul Immunol Inflamm;12:311-5. doi: 10.1080/092739490500354; PMid:15621870 